\title{
Executive Functions: Definition; Contexts and Neuropsychological Profiles
}

\author{
Dott Giulio Perrotta* \\ Department of Criminal and Investigative Psychology UNIFEDER, Italy
}

*Corresponding author: Dott Giulio Perrotta, Psychologist specializing in psychotherapy with a strategic approach; Forensic Criminologist expert in sectarian cults; esoteric and security profiles; Director of the Department of Criminal and Investigative Psychology UNIFEDER; SSPL Jurist; Teacher; CT/P; International Essayist, Italy.

Received Date: July 07, 2019

Published Date: August 14, 2019

\begin{abstract}
Starting from the concept of "executive functions"; we proceeded to describe the object under examination in a more complete and functional way; and their possible role in neuropsychology and developmental psychopathology. The use of statistical methods and clinical observation have allowed us to emphasize the understanding of the multi-componential structure of the processes themselves. Paying particular attention to the psychopathological contents listed in the DSM-V psychiatric manual; the main pathological forms were then analyzed to better contextualize the present discussion; also from a psychotherapeutic point of view; with the preferential choice of the strategic approach for the management of pathological disorders related to the deficit of executive functions.
\end{abstract}

Keywords: Psychology; Neuroscience; Executive functions; Developmental disorders; Amygdala; Prefrontal cortex; Cerebellum; Limbic system; Psychotherapy; Psychopharmacology; Strategic approach

\section{Introduction}

\section{Definition and clinical context of executive functions}

The term "executive functions" (E.F.) refers to a complex cognitive construct in the form of a system organized in functional modules of the mind; where a series of processes necessary to maintain an appropriate; organized and flexible planning mode are included. resolution (or problem solving); control and coordination; aimed at a purpose [1-3].

To simplify; the executive functions are those abilities that answer the question $<<$ Who is in charge? $>>$ and concern mental processes aimed at the elaboration of adaptive cognitive-behavioral schemes; at the base of planning; decision making; working memory; corrective response to error feedback; predominant habits; mental flexibility.

This complex construct thus implies:

a. An ability to inhibit a response or to postpone it at a later and more appropriate time.

b. A strategic and flexible planning of behavioral sequences.

c. A mental representation of the task that includes both the relevant information encoded in the memory and the future objectives to be achieved.

There are several possible definitions; from a theoretical and organizational point of view [4]:

1) The executive functions represent a system of abilities that allows to create objectives; to preserve them in memory; to control the actions; to foresee the obstacles and to reach objectives (Stuss 1992).

2) Executive functions represent the set of abilities that allow the person to successfully implement independent; intentional and useful behaviors (Lezak 1993).

3) Executive functions are higher-order cognitive functions that make it possible to formulate objectives and plans; remember these plans over time; choose and start actions that allow us to reach those goals; monitor the behavior and adjust it so as to arrive at those goals (Aron 2008).

It therefore appears evident that the executive functions are not easy to define; since this term does not refer to a single capacity but rather to a set of different sub-processes necessary to perform 
a specific task. They are superior cortical functions responsible for the control and planning of behavior; they are processes that allow the person to plan and implement projects aimed at achieving a goal and are necessary because they guarantee the monitoring and modification of their behavior in case of need or they adapt it to new contextual situations.

They consist of six steps:

1) Analyzing the task.

2) Plan how to achieve the task.

3) Organize the steps needed to carry out the task in question.

4) Develop a timeline to complete the task.

5) Adjust or change steps; if necessary; to complete the task

6) Complete the task in a timely manner.

They are therefore executive functions surely:

a) The inhibition; or the ability to focus attention on the relevant data ignoring the distractors and inhibiting inadequate or impulsive motor and emotional responses with respect to stimuli.

b) Flexibility; or the ability to move from one set of stimuli to another based on information from the context.

c) Planning; or the ability to formulate a general plan and organize actions in a hierarchical sequence of goals.

d) The working memory; or the ability to activate and maintain the plan and the work area at a mental level; to have a mental reference set on which to work mentally.

e) Attention; or the ability to maintain concentration on a given element.

f) Fluency; or the ability of divergent thinking and ability to generate new and different solutions with respect to a problem [5].

Although those most investigated for information on cognitive functioning are the basic functions of working memory [6] (or updating; the ability to maintain; update and process information in mind in time for the resolution of a task); of cognitive flexibility (or shifting; the ability to pass from one mental operation to another by controlling the mutual interference between the two actions) and inhibition (or inhibition; the ability to control automatic responses that interfere in achieving of a purpose); the executive domain does not end with the only cognitive processes listed above; but also involves mechanisms that play a part in the regulation of emotions; behavior and motivation.

A dichotomous distinction has been formulated in recent years between "Hot" executive functions and "Cool" executive functions [Zelazo 2004]:

a) The "Cool" executive functions represent those functions based on a complex; cognitive; controlled and slower processing; which are activated when the subject is dealing with abstract and decontextualized problems. The neurophysiological area used for these functions is the dorso-lateral prefrontal cortex [7].

b) The "Hot" executive functions are linked to an automatic and emotional processing of the stimuli; or a simple and rapid programming that intervenes in situations of stress; these functions are required in significant situations and involved in the regulation of emotion and motivation. The neurophysiological area used for these functions is the ventromedial prefrontal cortex [8].

The "Hot" executive functions and the "Cool" executive functions; according to this theoretical construct; work synchronously in order to guarantee an ideal functioning; but neuropsychological studies suggest a double dissociation between the two types of functions; documenting injuries on load Hot in the absence of problems against the Cool and the Cool in the absence of Hot.

The advances in the methodological field and in particular the conception of experimental tests with motor; linguistic and memory requirements; compatible with the level of competence of the child in early childhood; have allowed us to observe how the development of executive functions begins earlier; compared to what was previously assumed; this applies to both the Cool and the Hot ones. As far as the Cool is concerned; we have already seen how at 12 weeks the child is able to preserve the memory of the objective structure of an event in which he was the protagonist to reuse it in similar situations; from seven to eight months the first signs of working memory and inhibitory control begin to appear; regarding the Hot; some observations seem to suggest difficulties in the control of this executive domain in the first two years of life; although the processes of cortical development seem to affect these regions before those involved in the Cool: the child would indeed have difficulty in regulating the emotions and in postponing rewards/gratifications and presenting a way of relating to the self-centered world. Between the ages of three and five; the child succeeds in tasks that require maintaining information in the mind and at the same time the capacity for inhibition; between three and four years the ability to generate concepts develops; between four and five years the attentional control matures and there is an improvement in cognitive flexibility and in the ability to formulate strategies; at five years there is an increase in working memory and therefore in the ability to temporarily preserve and manipulate information online.

With preadolescence some executive skills reach maturity. Between seven and eight years and between nine and twelve years there is an increase in sensitivity to feedback in problem-solving; in the formulation of concepts and in the control of impulsiveness. At the age of seven; considerable progress has been reported in the speed of execution; in the ability to use the strategies; in the ability to maintain information in the mind and to work with it. Between the ages of eight and ten adult levels are reached in cognitive flexibility and at ten years the ability to maintain the set; the verification of hypotheses and impulse control is manifested; there 
is an improvement in the inhibitory control; in the vigilance and in the attention sustained between the eight and eleven years; period in which besides is assisted to an improvement in the performances tests that conjugate inhibitory competences and working memory; this last one suffers further efficiency improvements between nine and twelve years.

An improvement in the ability to understand emotions; intentions; beliefs and desires is noted in this period. Between thirteen and fifteen years there is an increase in memory strategies; in its efficiency; in time planning; in problem-solving and in the search for hypotheses. Furthermore; verbal fluency and the ability to plan complex motor sequences mature at the age of twelve. The changes of this period; both on the cognitive and the executive side; allow the person to cope with the new and growing demands that the physical and social environment put on him; experiencing a sense of independence; responsibility; and social awareness.

At fifteen years there is an improvement as regards attentional control and processing speed as well as maturation in inhibitory control. Between the ages of sixteen and nineteen; progress is made in working memory; problem-solving and strategic planning. From an executive point of view; Hot improves the ability to make decisions in the presence of rewards and losses. Between the ages of twenty and thirty; working memory; planning; problem-solving and the ability to implement targeted behaviors reach higher levels of functioning.

As regards the Hot Executive functions; the achievement of mature decision-making levels is achieved. With aging there is a gradual deterioration in some cognitive areas including the Executive Functions; although some changes are not evident before eighty years even though the brain degenerative process begins in the third decade of life. Between the ages of thirty and forty-nine; there is a decrease in information storage and temporal sequencing skills; the ability to formulate concepts; organization; planning and attentional shifting worsen between the fifty-three and sixty-four years. Starting from the age of sixty-five; memory difficulties are reported [9-11].

It is good to underline however that even today we think of the executive system and the attention system as separate entities: attention would act on sensory information and on internal representations while the executive system on behavior. If the attentional aspects allow the executive functions to mature; the executive system is seen as a form of attention directed towards oneself. Therefore, the development of E.F. involves a consolidation of intellectual cognitive abilities; learning and memories.

\section{The Neural Correlates in Eating Disorders [12]}

If neuroscience has been trying for decades to associate specific brain functions with specific brain areas; with the relative recent advent of functional neuroimaging and brain mapping; this commitment seems to have become dominant; however; the debate on the selective localization of complex faculties remains open; despite their historical location; identified in the so-called frontal lobe syndrome; even preceded the formulation of the construct (Galati; Tosoni; 2010); in fact; traditionally; they were classified as executive disorders those following damage in the prefrontal cortex.

Recent studies of neuroimage (Galati; Tosoni; 2010); carried out on healthy people through classical neuropsychological tests for the examination of E.F. reveal; however; also the activation of the posterior parietal cortex and of various subcortical centers; in addition to the areas of prefrontal cortex; whose functional subdivisions; however; still remain difficult to identify; due to its anatomy and its heterogeneous functionalities. In particular; the studies show that the frontal lobes are functionally connected with: the posterior parietal cortex; which appears to be involved in the reconfiguration of the responses and in the behavioral modifications (Sohn et al; 2000; Barber and Carter; 2005); the basal ganglia; the anterior cingulate; which seems particularly involved in situations of control of cognitive conflicts between environmental stimuli or behaviors and in the selection of agent responses in case of uncertainty (Carter and Van Veen; 2007; Rushworth and Beherens; 2008).

The scholars of the Executive Functions; therefore; remain cautious about their specific localization; preferring to believe that they are implemented in multiple distributed circuits; each of which includes connections with some portion of the prefrontal cortex (Galati; Tosoni; 2010; p. 36.). The same Luria (1962) who; on the basis of numerous clinical observations; first theorized the existence of a central control system for some higher order functions; such as planning; monitoring; self-regulation; involved more involvement interconnected cortical and subcortical areas: prefrontal cortex; cerebellum; some subcortical nuclei.

Therefore; the study on the functions performed by the prefrontal cortex remains open. It is believed that the executive functions are anatomically related to different areas of the prefrontal cortex; and to the associated cortico-subcortical circuits:

a) The dorso-lateral prefrontal area would be particularly involved in the abstraction and planning of actions.

b) The orbital-frontal area would be involved in the regulation of emotions and decision-making processes.

c) The anterior cingulate area (especially in the dorsal part) would be involved in the control of motivation and interfering stimuli.

The empirical evidences derived from the neuropsychological approach and from the neuroimaging show however that the executive functions connected to the orbito-frontal cortex mature early with respect to the executive functions connected to the dorso-lateral pre-frontal cortex [13]. An interesting review of 2006 takes in detail; albeit dated; the cognitive; affective and behavioral correlates of brain maturation that occurs during adolescence. This maturation; due to phenomena of myelination and synaptic pruning; is particularly accentuated in the prefrontal cortex; the 
main site of decision-making processes. If the executive functions and the decision-making processes are based on the functioning of prefrontal areas; which change considerably during adolescence; it can be hypothesized that the decision-making abilities of adolescents are still immature, and this may explain their risky behavior. This hypothesis is discussed; also, in relation to the onset of psychopathological disorders in this age group [14].

\section{The Clinical and Strategic Approach in the Management of Executive Function Deficits [15]}

Following an injury or dysfunction in the frontal lobes; due for example to a head injury; a degenerative pathology or a neoplasm; the patient can show the symptoms of what is called "frontal syndrome". Frontal syndrome is a clinical picture characterized by cognitive deficits and / or behavioral; emotional and motor disorders.

Studies on adult patients with lesions in different areas of the prefrontal cortex show; in fact; partially different neuropsychological pictures:

1) Lesions in the anterior orbital part; in general; cause personality modifications and disinhibition.

2) The lesions in the orbito-frontal part; also because it is closer to the amygdala; to the hippocampus and to the hypothalamus; areas that mediate between internal states and environmental stimuli; generally present inattentive; impulsive behaviors; difficulty in problem solving and in taking of decisions; serious antisocial conduct.

3) Lesions in the medial part; including the anterior cingulate gyrus; cause poor motor control and difficulty in maintaining focused attention.

4) The laterals of the prefrontal cortex present action planning disorders; especially related to the management of mental representations useful for achieving a purpose and the difficulties related to written and spoken language are understood.

The lesions of the frontal cortex; caused by expansive processes of tumor; vascular or hypoxic origin; or cranial traumas or degenerative processes of the nervous system; therefore, lead to deficits in executive functions; in particular problems:

a) In planning and problem solving: the person has difficulty planning and executing a sequence of actions to reach a goal; but also in the planning of sequences of movements.

b) In cognitive flexibility: it has a rigid; non-flexible behavior and puts into effect perseverances; always giving the same answer or using the same strategy even when it proves inadequate.

c) In the working memory: the memory disorders that can be classified in the frontal amnesic syndrome are characterized by the inability to retain new information; greater distractibility and confabulations; difficulty in using memorization strategies; inability to know how to use the new acquired data; incapacity to memorize voluntarily.

d) In the inhibition of automatic behaviors not congruent with the situation: it is the case of the "environmental syndrome" or "dependence of use": placed for example in front of objects that it is used to use; the person uses them without any invitation and without any reason (for example; a patient who in front of a bottle of water placed on the examiner's desk; takes it and drinks it).

e) In decision-making: the difficulty of deciding in an advantageous way for oneself and of respecting social norms is understood (Bechara et al. 2000; Rolls; 2000). Patients with this disorder are more likely to make risky choices and develop; for example; a gambling addiction.

f) In the regulation of emotions and behavior: we can have a patient who shows a picture of disinhibited type symptoms characterized by euphoria; restlessness; sexual disinhibition; inappropriate social behavior; poor interest in others; uninhibited behavior; with little impulse control; easy irritability and aggressiveness; euphoria; emotional lability; or an apathetic type symptomatology with a "pseudo-depressed" personality; therefore with modifications characterized by indifference; apathy; decreased spontaneity; reduced sexual interest; reduced expression of emotions; decreased verbal productivity (including mutism); decreased motor behavior.

g) Low self-criticism and judgment: the person have a deficit in judging reality; especially when the situation is new or complex and a lack of critical attitude towards the actions carried out. It also shows difficulty in correcting its errors and inability to modify or schedule new behaviors.

In some syndromes then there are deficits in executive functions; such as in autism and dyslexia; Executive deficits have been detected in attention deficit / hyperactivity disorder (ADHD); schizophrenia and conduct disorder. It is thought that many mental disorders are associated with this type of deficit; although in every disorder it is likely to change the degree to which each component of executive functions is involved. Deficits related to executive functions can be manifested in behavioral symptoms such as: environmental dependence syndrome; with use behavior; that is; as soon as the subject notices an object that he is used to using in a certain way; he uses it; even if the context would require a inhibition of this behavior (eg the subject goes to the doctor and as soon as he notices the window opens it; without a precise reason); and imitation behaviors; that is; the subject spontaneously imitates the gestures of the person in front of him; hypoactivity; such as apathy or anhedonia (in which the person does not perform behaviors that would also give him gratification (such as activities related to food or affective activities); hyperactivity; ie distractibility; impulsiveness; disinhibition. In general; the behavior may appear disorganized [16]. 
Compared to the diagnosis; however; one mustrely on a series of neuropsychological tests; including the Trail making test (versions A and B). The other tests that are most used for the evaluation of the executive functions are: the Tower of London that proves the ability of planning; problem solving and inhibition; the dimentional change card sort test; which is a task that evaluates flexibility; the matching familiar figure test that evaluates the use of visual search strategies; control of the impulse response and interference [17].

\section{Conclusion}

The E.F. they represent an important field of research and clinical work in the field of cognitive developmental neuropsychology. There are numerous attempts to summarize the common characteristics of the skills related to E.F.: the ability to inhibit overbearing responses and to organize behavior based on arbitrary rules [18]; the ability of the "central executive" to select the appropriate schemes of action from a repertoire activated by specific inputs [19].

The hypothesis of the multidimensionality of FEs [20-22] is increasingly accepted and several studies are highlighting the evolutionary trajectories of the different subdomains in the typical development; starting to outline a staged process with a complex hierarchical organization. In the clinical field the impairments of the E.F. they are related to numerous cognitive and behavioral difficulties: limited sustained attention; perseverative responses; impaired initiation of actions; poor use of feedback; difficulties in planning and organization; problems related to the storage and manipulation of mental representations. Furthermore; studies on the impairment of E.F. in neurodevelopmental disorders.

These include autism and DGS; in which the E.F. they appear to be pervasive in different domains; severe and pre-existing over time. Other developmental disorders seem to show a more specific profile; with the impairment of some subdomains: the A.H.D.; with the deficit in inhibition; represents one of the most studied cases. For other disorders the research results are more controversial. The differences in the profiles of the E.F. in these disorders they support the hypothesis of a fraction ability of E.F. in subdomains; at least partially independent. It should also be specified that the various subdomains have not been fully studied in all the disorders. Despite these limitations; at least partially specific "executive profiles" of various disorders are emerging [23].

A greater knowledge of these profiles and their evolutionary trajectories; combined with an attention to "ecological validity"; can allow us a greater understanding of the mental functioning of children with typical and atypical development; with potential relapses in the diagnostic and therapeutic-rehabilitative field [2426].

\section{Acknowledgement}

None.

\section{Conflict of Interest}

No conflict of interest.

\section{References}

1. Luria AR (1996) Higher cortical functions in man. Basic Books, New York, USA.

2. Shallice T (1988) From neuropsychology to mental structure. Cambridge: University Press, USA.

3. Welsh MC, Pennington BF (1988) Assessing frontal lobe functioning in children: views from developmental psychology. Developmental Neuropsychology 4(3): 199-230.

4. Cantagallo A, Spintoni G, Antonucci G (2010) Le Funzioni Esecutive. Carocci Editore, Italy.

5. Marzocchi GM, Valagussa S (2011) Le Funzioni Esecutive in età evolutiva. Franco Angeli, France.

6. Baddeley A (1992) Working memory. Science 255(5044): 556-559.

7. McKay Moore Sohlberg, Catherine A Mateer (2017) Cognitive rehabilitation: an integrative neuropsychological approach. Guilford Publications, USA

8. Sumowski JF, Rocca MA, Leavitt VM, Dackovic J, Mesaros S, et al. (2014) Brain reserve and cognitive reserve protect against cognitive decline over 4.5 years in MS. Neurology 82(20): 1776-1783.

9. Diamond A, Briand L, Fossella J, Gehlbach L (2004) Genetic and neurochimical modulation of Prefrontal cognitive functions in children. Am J Psychiat 161(1): 125-132.

10. Fuster JM (1997) The prefrontal cortex: anatomy, physiology, and neuropsychology of the frontal lobe, ( $3^{\text {rd }}$ edn.), Philadelphia: LippincottRaven, USA.

11. Alvarez JA, Emory E (2006) Executive function and the frontal lobes: a meta-analytic review. Neuropsychology Rev 16(1): 17-42.

12. Bettcher BM, Mungas D, Patel N, Elofson J, Dutt S, et al. (2016) Neuroanatomical Substrates of Executive Functions: Beyond Prefrontal Structures. Neuropsychologia 85: 100-109.

13. Blakemore SJ, Choudhury S (2006) Development of the adolescent brain: implications for executive function and social cognition. J Child Psychol Psychiatry 47(3-4): 296-312.

14. DC Miller, DE Maricle (2018) Essentials of School Neuropsychological Assessment. ( $3^{\text {rd }}$ edn.), USA.

15. G Perrotta (2019) Psicologia clinica. In: Luxco (edt.), (1ºdn.), USA.

16. Ozonoff S, Jensen J (1999) Brief report: specific executive function profiles in three neurodevelopmental disorders. J Aut Dev Disord 29: 171-177.

17. Seguin JR, Boulerice B, Harden PW (1999) Executive Functions and physical aggression after controlling for attention deficit disorder, general memory and IQ. J Child Psychol Psychiat 40: 1197-1208.

18. Anderson P (2002) Assessment and development of executive function (EF) during childhood. Child Neuropsychol 8(2): 71-82.

19. Brookshire B, Levin HS, Song J, Zhang L (2004) Components of executive function in typically developing and head-injured children. Dev Neuropsychol 25(1-2): 61-83.

20. Miyake A, Friedman NP, Emerson MJ, Witzki AH, Howerter A, et al. (2000) The unity and diversity of executive functions and their contributions to complex frontal lobe tasks: a latent variable analysis. Cognit Psychol 41(1): 49-100.

21. Fisher N, Happé F (2005) A training study of Theory of Mind and Executive Function in children with autistic spectrum disorders. J Aut Dev Disord 35(6): 757-771.

22. Marlowe WB (2000) An intervention for children with disorders of executive functions. Dev Neuropsychol 18(3): 445-454

23. Stievano P, Valeri G (2006) Funzioni esecutive in età prescolare: ipotesi abilitative in un bambino a rischio. Psichiat Inf Adol 73: 417-431. 